\title{
A new Technique for Position Control of Induction Motor Using Adaptive Inverse Control
}

\author{
Aamir Hashim Obeid Ahmed \\ Electrical Engineering Department \\ Sudan University of Science and \\ Technology \\ Khartoum, Sudan \\ aamirahmed@sustech.edu
}

\author{
Martino O. Ajangnay \\ Electrical Engineering Department \\ Sudan University of Science and \\ Technology \\ Khartoum, Sudan \\ Ajangnay16@hotmail.com
}

Shamboul A. Mohamed

Dean College of Engineering

Sudan University of Science

and Technology

Khartoum, Sudan
Matthew W. Dunnigan

Electrical, Electronics and

Computer Engineering

Heriot-Watt University Edinburgh, UK

m.w.dunnigan@hw.ac.uk

\begin{abstract}
Control of Induction Motor (IM) is well known to be difficult owing to the fact the models of IM are highly nonlinear and time variant. In this paper, to achieve accurate control performance of rotor position control of IM, a new method is proposed by using adaptive inverse control (AIC) technique. In recent years, AIC is a very vivid field because of its advantages. It is quite different from the traditional control. AIC is actually an open loop control scheme and so in the AIC the instability problem cased by feedback control is avoided and the better dynamic performances can also be achieved. The model of IM is identified using adaptive filter as well as the inverse model of the IM, which was used as a controller. The significant of using the inverse of the IM dynamic as a controller is to makes the IM output response to converge to the reference input signal. To validate the performances of the proposed new control scheme, we provided a series of simulation results.
\end{abstract}

\section{INTRODUCTION}

Only separately excited DC motors were previously used in most high performance speed and/or position control applications since torque and motor flux could be controlled easily and independently. However, DC motors have basically two drawbacks, which are the existence of commutators and brushes. These two disadvantages implied not only periodic maintenance but also difficulty to work in dirty and explosive environments; difficulty that sometimes used to become in impossibility. On the other hand, induction motor is robust, easily maintained and reliable [1-3] Moreover, because of the advances in power electronics and microprocessor, induction motor drives used in variable speed and position control have become more attractive in industrial processes such as robot manipulators, factory automations and transportation applications. However, it is known that the control of induction motor is relatively difficult compared to the kinds of motors, such as DC motors. In fact, the induction motor presents a complex nonlinear system with parametric variation. In the last few decades, abundant research and development efforts for induction motor control technology have been made [4-6]. Among them, the Field Oriented Control (FOC) or Vector Control (VC) is the most popular one. The FOC for induction motor was introduced for the first time by Blaschke in the early 1970s. The FOC technique guarantees the decoupling of torque and flux control commands of the induction motor, so makes the induction motor be controlled linearly as a separated excited DC motor [7-8]. However, the performance is sensitive to the variation of motor parameters, especially the rotor time constant, which varies with temperature and the saturation of the magnetizing inductance. To overcome the above problems and achieve accurate control performance of rotor position control of induction motor, a novel approach is proposed by using AIC technique. AIC is a novel approach which can make a plant track the input command signal with a controller whose transfer function approximate the inverse of the plant transfer function. Compared with traditional methods, AIC can achieve specified dynamic responses more easily and has better ability of disturbance rejection [9-11]. The key of AIC is how to construct inverse model of controlled system accurately.

The organization of this paper is as follows. In section II, the basic concept of AIC is briefly reviewed. Section III the background of adaptive filter is briefly reviewed. Section IV introduces the induction motor model used in the work and the new proposed technique is discussed. Section V, presents some simulation results on an induction motor with the new proposed technique. The last section contains the conclusion.

\section{BASIC CONCEPT OF AIC}

AIC is a very novel control technique for the design and analysis in the industry process control system. AIC was named and proposed by professor Widrow in 1986 [9], which do not require a precise initial plant model. AIC technique has been successfully applied to a variety of control problems. The control philosophy is feed forward but feedback is present by means of the adaptation loop of the controller weights. AIC suggests a controller in serial with controlled plant, and the control of the plant dynamics can be achieved by preceding the plant with an adaptive controller whose transfer function approximates the inverse of that of the plant. The objective of this system is to cause the plant output to follow the command input. In AIC the coefficients of the controller are adaptively adjusted by an adaptive algorithm which is controlled by the input signal and the error signal. The structure of AIC consists of three main parts. First, adapt a plant model using adaptive system techniques. Second, need to calculate the inverse model of the plant model and at last the inverse model will serve as a controller to control the plant [9-11].

\section{A. Adaptive system modelling}

Adaptive system modeling or identification had been widely applied in control system, communication, and signal processing. Figure 1 illustrates how this can be done with an adaptive filter. The unknown system (plant) is connected in 
parallel with an adaptive filter; where the modelling signal applied simultaneously to the adaptive filter and unknown system. Three major issues are involved in adaptive system identification: The excitation signal, the filter structure, and the adaptation mechanism. The optimal model of the plant was obtained by adapting the weights or coefficients of an adaptive filter so that the mean square error between the output of the plant and adaptive filter output is minimized.

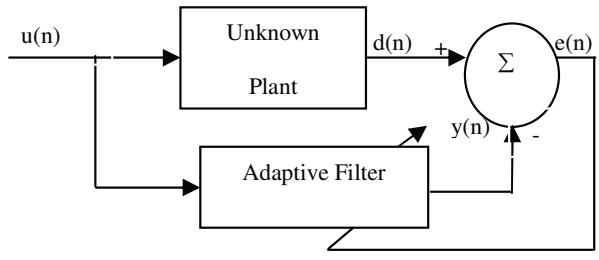

Fig. 1 System identification using adaptive filtering

\section{B. Adaptive inverse plant modeling}

Adaptive filter technique is also used in modeling to calculate the inverse model of the plant. The plant generally has poles and zeros. The inverse of the plant therefore should have zeros and poles. This technique can be used to form the inverse model of minimum-phase plant as well as non-minimum-phase. For example, if the system under investigation is known to be minimum phase, that is, has all of its zeros inside the unit circle in the z-plane, then the inverse will be stable with all its poles inside the unit circle When the plant is non-minimum-phase, then some of the poles of the inverse will be outside the unit circle and the inverse will be unstable. In the case of unstable plant, conventional feedback technique should be applied to stabilize it. Then the combination of the plant and its feedback stabilizer can be regarded as an equivalent stable plant [11]. The inverse of the plant model can be achieved by placing the adaptive filter at the same path with the plant as shown in Figure 2 below. The plant input is its command signal. The plant output is the input to adaptive filter.

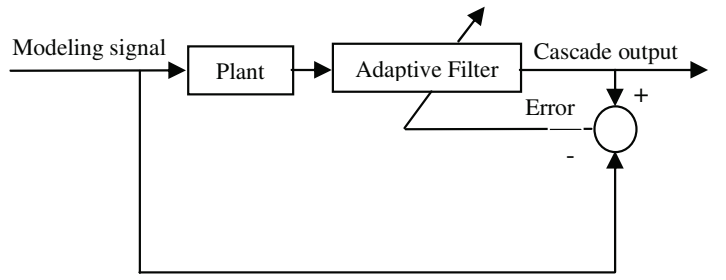

Fig. 2 Inverse plant model

The adaptive algorithm attempts to make the cascade of the plant and adaptive inverse behave like a unit gain. This process is often called deconvolution [9].

\section{ADAPTIVE FILTERS}

Adaptive filters have received considerable attention by researchers over the past 25 years. As a result, many adaptive filter structures and adaptation algorithms have been developed during this period. The theory of adaptive filtering is fundamental to AIC. There are two fundamental types of digital filters: Finite Impulse Response (FIR) and Infinite Impulse Response (IIR). An important advantage of the FIR model of IIR model is that the FIR filters always stable. The FIR filter is also called an all zero system, because the weight vector only defines the zeros of the filter whereas the filter's poles all lie at the origin of the unit circle. Furthermore, an adaptive FIR filter is many times preferred over an adaptive IIR filter due to its simplicity and robustness. The adaptive IIR filter generally provides better performance than FIR filter that has the same number of coefficients [12-13].

The adaptive filter consists of two stages, filtering and adaptation. The filtering stage involves computation of output and generation of estimation error by comparing this output with the desired response. In the adaptive stage the tap weight vectors of the FIR filter are adjusted such that estimation error decreases with the each iteration. The key component of an adaptive filter is the adaptation algorithm, which is the method to determine the filter coefficients from the available data. The performance of these adaptive algorithms is highly dependent on their filter order and signal condition. Furthermore, the choice of an adaptive algorithm for any given application is determined by both costs of implementation and performance, with higher cost usually paid for improved performance. There are two different types of adaptation algorithms: a priori and a posteriori, which is based on the difference in coefficient updating methods. When the desired response is estimated using the previous coefficient matrix then it is called a priori. When the estimate is derived using the current coefficient matrix it is called a posteriori. We have used the a priori method for desired response prediction because it is more direct and easier to implement. For FIR adaptive filtering, the most widely adaptive algorithms for updating the filter weights are the Recursive Least Squares (RLS), and Least Mean Squares (LMS) or its normalized version.

\section{A. The LMS Algorithm}

The LMS algorithm, which was first proposed by Widrow and Hoff in 1960, is the most widely used adaptive filtering algorithm in practice [14]. The LMS algorithm belongs to the family of stochastic gradient linear adaptive filtering algorithm. It is called a stochastic gradient algorithm because it iterates each tap weight in the direction of the gradient of the squared magnitude of the error signal. Although in the subsequent four decades numerous alternative adaptive algorithms have been proposed, it is still one of the most efficient algorithms due to its simplicity of implementation, adaptation robustness, and low computational cost [12]. However, it suffers from a slow rate of convergence and high sensitivity to non stationary environments. Furthermore, its implementation requires the choice of an appropriate value for the step-size that affects the stability, steady-state mean square error (MSE), and convergence speed of the algorithm. 
For the each iteration the three basic equations governing the operation of the LMS algorithm are listed as follows [15]:

$$
\begin{aligned}
& y(n)=w(\mathrm{n})^{\mathrm{T}} u(\mathrm{n})=u(\mathrm{n})^{\mathrm{T}} w(\mathrm{n}) \\
& \mathrm{e}(\mathrm{n})=\mathrm{d}(\mathrm{n})-\mathrm{y}(\mathrm{n}) \\
& w(\mathrm{n}+1)=w(\mathrm{n})+2 \mu \mathrm{e}(\mathrm{n}) u(\mathrm{n})
\end{aligned}
$$

where $u(n)$ is the input when time is $n, w(n)$ is a weight vector, . $w(n+1)$ is a update of $w(n), e(n)$ is the error between desired signal d(n) and the filter output $y(n)$, and $\mu$ stands for step size that effects stability of adaptation and speed of convergence. Usually, the initial values in weight vector $\mathrm{w}(0)$ are set to zero. Selection of a suitable value for $\mu$ is imperative to the performance of the LMS algorithm, if the value is too small the time the adaptive filter takes to converge on the optimal solution will be too long; if $\mu$ is too large the adaptive filter becomes unstable and its output diverges [15-16].

\section{B. The Normalized LMS (NLMS) Algorithm}

One of the primary disadvantages of the LMS algorithm is having a fixed $\mu$ for the every iteration. One approach to overcome this limitation has been to use the NLMS algorithm [12]. The NLMS algorithm, an equally simple, but more robust variant of the LMS algorithm, exhibits a better balance between simplicity and performance than the LMS algorithm, and has been given more attention in real time applications. Furthermore, it possesses many advantages over the LMS algorithm; including having a faster convergence speed and providing for an automatic time-varying choice of the LMS step size parameter that affects the stability, and steady-state MSE. For the each iteration of the NLMS algorithm, the filter tap weights of the adaptive filter are updated according to the following steps:

$$
\begin{aligned}
& y(n)=w(\mathrm{n})^{\mathrm{T}} u(\mathrm{n})=u(\mathrm{n})^{\mathrm{T}} w(\mathrm{n}) \\
& \mathrm{e}(\mathrm{n})=\mathrm{d}(\mathrm{n})-\mathrm{y}(\mathrm{n}) \\
& w(\mathrm{n}+1)=w(n)+2 \frac{\mu}{\gamma+u^{T}(n) u(n)} u(n) e(n)
\end{aligned}
$$

Where $\gamma$ is a small positive constant in order to avoid division by zero when the values of the input vector are zero or close to it, the instability due to division by zero is avoided. The parameter $\mu$ is a constant step size value used to alter the convergence rate of the NLMS algorithm. Theoretically, it is within the range of $0<\mu<2$ for stable adaptation, however a more practical step size for NLMS is always less one unity.

\section{The RLS Algorithm}

Compared to the LMS and NLMS algorithms, the RLS algorithm has the advantage of faster convergence and small steady state error but this comes at the cost of increasing the complexity. Hence, the RLS algorithm requires longer computation time as well as a higher sensitivity to numerical instability. These disadvantages make the RLS algorithm unsuitable when a large number of taps is required for modelling. To implement the RLS algorithm, the following steps are executed in the following order [17].

$$
\begin{aligned}
& y(n)=w(\mathrm{n})^{\mathrm{T}} u(\mathrm{n})=u(\mathrm{n})^{\mathrm{T}} w(\mathrm{n}) \\
& \mathrm{e}(\mathrm{n})=\mathrm{d}(\mathrm{n})-\mathrm{y}(\mathrm{n}) \\
& w(\mathrm{n})=w(n-1)+\frac{P(n-1) u(n) e(n)}{\lambda+u^{T}(n) P(n-1) u(n)} \\
& P(\mathrm{n})=\left[P(n-1)-\frac{P(n-1) u(n) u^{T}(n) P(n-1)}{\lambda+u^{T}(n) P(n-1) u(n)}\right] / \lambda
\end{aligned}
$$

Where $\mathrm{P}(\mathrm{n})$ is the covariance matrix. The algorithm is initialized by setting $\mathrm{P}(0)=\delta I$, where $\delta$ is a small positive constant number, and $I$ is the identity matrix. The initial value $\mathrm{P}(0)$ can not be zero because it will remain zero. The parameter $\lambda$ is a positive constant which is less than or equal to unity and generally has a value near 0.99 . It is often referred to as the forgetting factor, as it controls the effective length of the memory of the algorithm [18].

\section{Position Control of IM}

In this section, we show the designed procedure for the rotor position control of induction motor system which is under the control by AIC. The position control goal is to force the rotor position $\theta_{r}$ to track the desired rotor position reference $\theta_{d}$. For the position control system, the mechanical equation of an induction motor drive can be represented as:

$T_{e}=J \ddot{\theta}_{r}+B \dot{\theta}_{r}+T_{L}$

Where $\mathrm{J}$ is the total mechanical moment inertia constant, $\mathrm{B}$ is the total damping coefficient, $T_{L}$ is the torque of external load disturbance, and Te denotes the electromagnetic torque. The $\mathrm{T}_{\mathrm{e}}$ can be defined as:

$T_{e}=K_{t} i_{q s e}^{*}=\frac{3 P L_{m}^{2}}{2 L_{r}} i_{d s}^{*} e^{i_{q s e}^{*}}$

Where $\mathrm{L}_{\mathrm{r}}$ is the rotor inductance and $\mathrm{L}_{\mathrm{m}}$ is the mutual inductance, $\mathrm{P}$ is the number of pole pairs, $i_{\mathrm{qse}}^{*}$ and $i_{\mathrm{dse}}^{*}$ denote the torque and flux current commands. For induction motor modeling and inverse induction motor modeling we used the LMS, NLMS, and RLS algorithms. After the induction motor model is completed, the inverse of the induction motor modeling can be achieved by placing the adaptive filter at the same path with the induction motor model. After the controller is established, we can cascade it with the induction motor model to track the desired reference signal as shown in Figure 3.

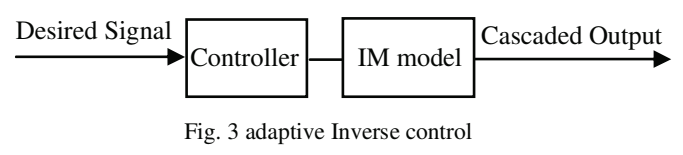

\section{Simulation RESUlTS}

To evaluate the effectiveness and performance of the new AIC technique, extensive computer simulation results are presented to compare the performance of the new proposed control strategy under different types of adaptive algorithms. 
The figure of merit that is used to observe convergence speed of adaptive filters is the MSE. The rating and parameters of the IM system under consideration are given in Appendix. The simulation is carried out based on the schemes shown in Figure 4.

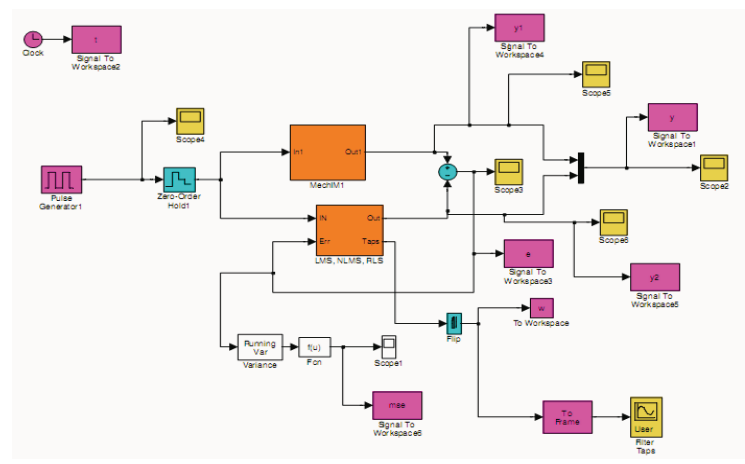

(a) Identification of induction motor

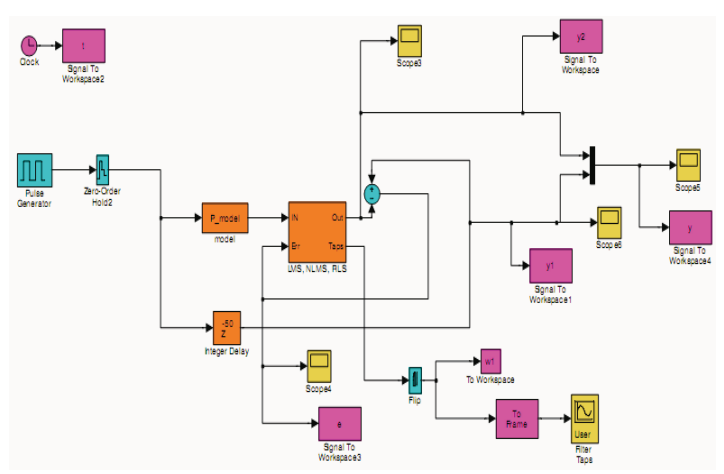

(b) Inverse model of induction motor

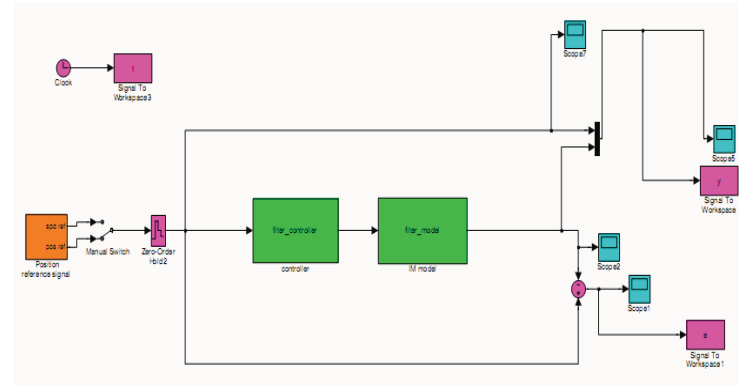

(c) Speed control of induction motor

Fig. 4 Simulation system setup

A. The LMS Algorithm Simulation Results

The FIR filter representing the induction motor modeled and inverse induction motor model has 64 taps with step size parameter value 0.01 . The MSE learning curve is shown in
Figure 5. The minimum mean square error (MMSE) obtained is $-85.2 \mathrm{~dB}$, and the LMS algorithm has the slowest convergence time amongst the filtering learning algorithms considered. In order to verify the robustness of the LMS algorithm against measurement noise, Gaussian zero mean white noise with the variance of $10^{-3}$ was added to the output of the unknown system. The MSE learning curve is shown in Figure 6 . The result shows that the convergence time more alters when the measurement noise is added. Figure 7 shows the induction motor tracking performance between desired and actual rotor position signals. Figure 8 shows the induction motor rotor position tracking error. The peak rotor position error between desired and actual rotor position signals is within the range $\pm 0.117 \mathrm{rad}$.

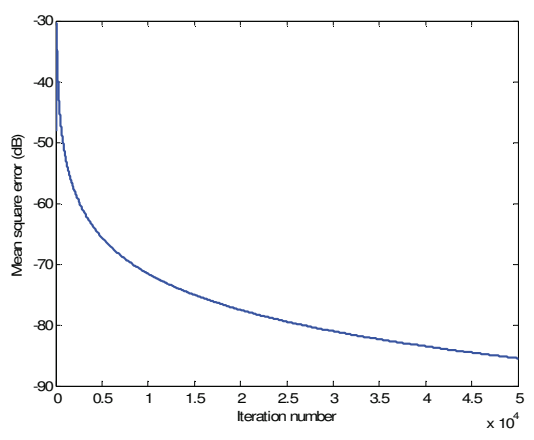

Fig. 5 MSE learning curve

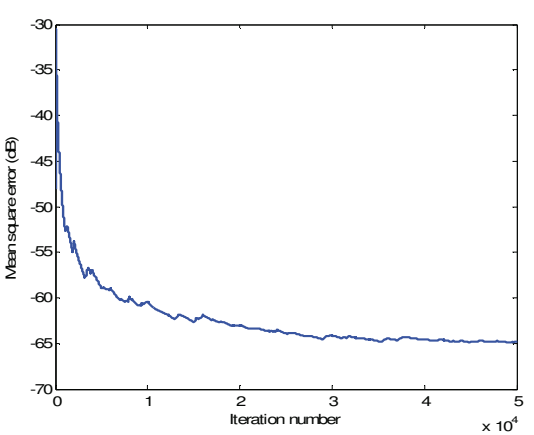

Fig. 6 MSE when noise added

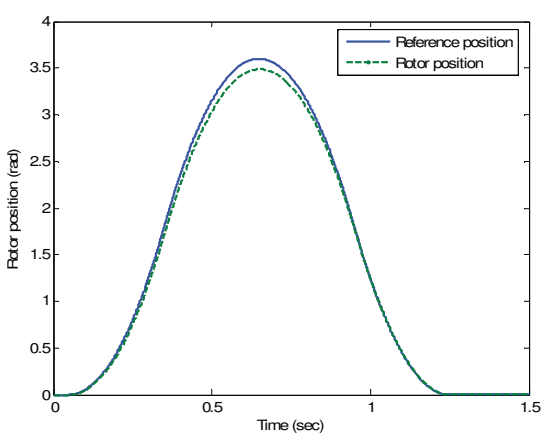

Fig. 7 Rotor position signals 


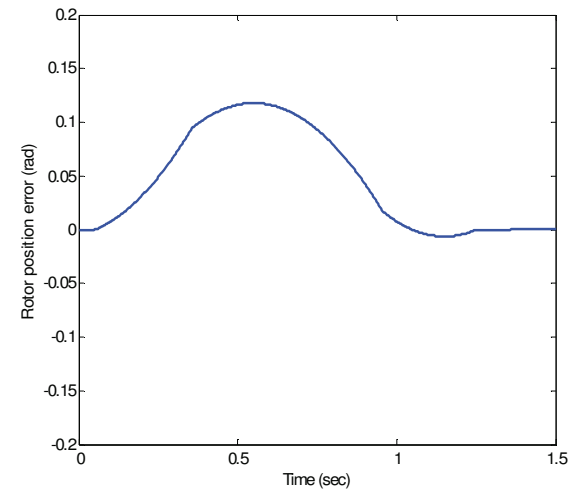

Fig. 8 Rotor position error

B. The NLMS Algorithm Simulation Results

The FIR filter representing the induction motor modeled and inverse induction motor model has 64 taps with step size parameter value 0.1. The MSE learning curve is shown in Figure 9, which indicates a MMSE of about $-80.1 \mathrm{~dB}$. This is figure shows that NLMS algorithm converges faster than standard LMS algorithm. In order to verify the robustness of the NLMS algorithm against measurement noise, Gaussian zero mean white noise with the variance of $10^{-3}$ was added to the output of the unknown system. The MSE learning curve is shown in Figure 10. Comparing Figure 6, and Figure 10, it is clear that the NLMS algorithm still converge faster than standard LMS algorithm when the measurement noise is added. The induction motor tracking performance between desired and actual rotor position signals is shown in Figure 11. Figure 12 shows the induction motor rotor position error. The peak rotor position error between desired and actual rotor position signals is within the range $\pm 0.043 \mathrm{rad}$. However, the NLMS shows better peak rotor position error compared to the LMS algorithm.

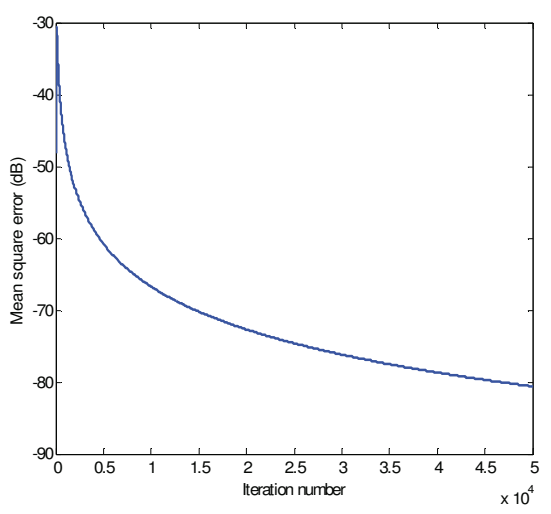

Fig. 9 MSE learning curve

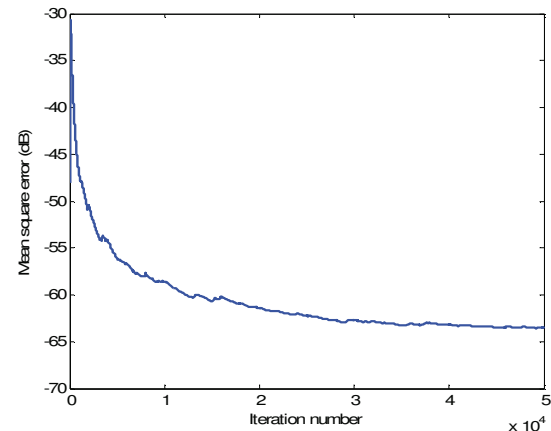

Fig. 10 MSE when noise added

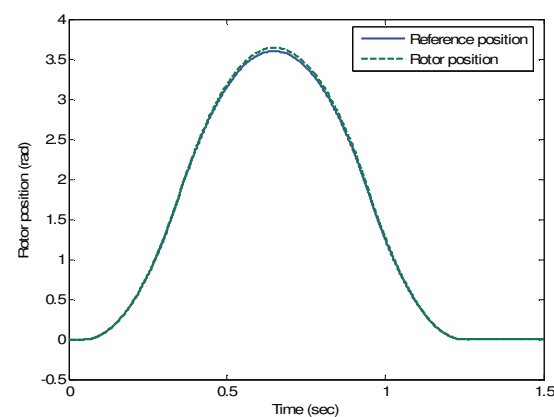

Fig. 11 Rotor position signals

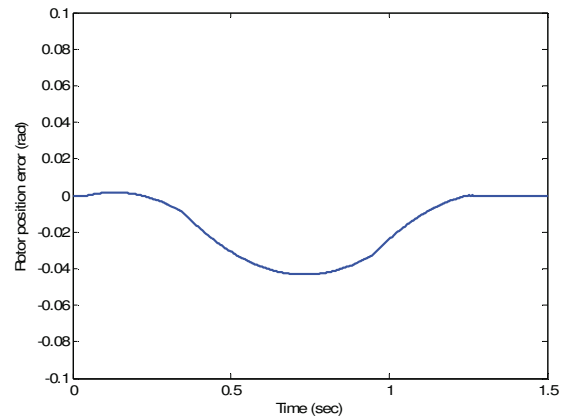

Fig. 12 Rotor position error

C. The RLS Algorithm Simulation Results

The FIR filter representing the IM modeled and inverse IM model has 16 taps. A greater number of taps is not suitable, because the memory requirement for the algorithm grows approximately with the square of the number of taps. For the RLS algorithm we have initialized the P-matrix with $\delta=0.04$ in order to get fast initial convergence. Figure 13 shows the MSE learning curve when the forgetting factor is set to 0.9999. Simulations show that the algorithm is sensitive to the choice of forgetting factor and it should be close to one. The RLS algorithm requires about 350 iterations to converge with a MMSE of about $-71.1 \mathrm{~dB}$. Although, the RLS algorithm has the advantage of having a faster convergence rate than the conventional LMS and NLMS algorithms, which means that the RLS algorithm model more accurately than the 
another two adaptive algorithms with fewer taps. In order to verify the robustness of the RLS algorithm against measurement noise, Gaussian zero mean white noise with the variance of $10^{-3}$ was added to the output of the unknown system. The MSE curve is shown in Figure 14. The result shows that the RLS algorithm is more robust than the other introduced adaptive algorithms and convergence time still better when the measurement noise is added. Figure 15 shows the induction motor tracking performance between desired and actual rotor position signals. The induction motor rotor position error is shown in Figure 16. The peak rotor position error between desired and actual rotor position signals is within the range $\pm 0.019 \mathrm{rad}$. Comparing Figures 8,12 , and 16, it is clear that the RLS produces smaller peak rotor position error than the standard LMS, and NLMS algorithms.

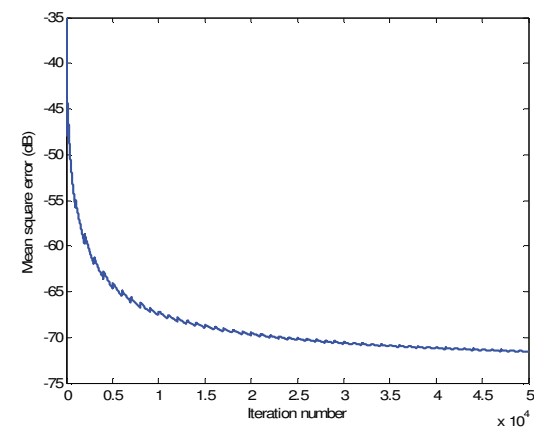

Fig. 13 MSE learning curve

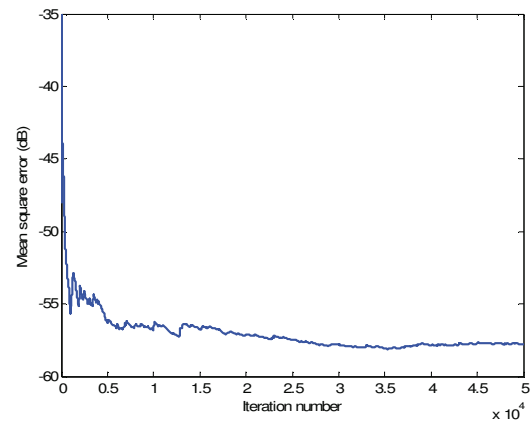

Fig. 14 MSE when noise added

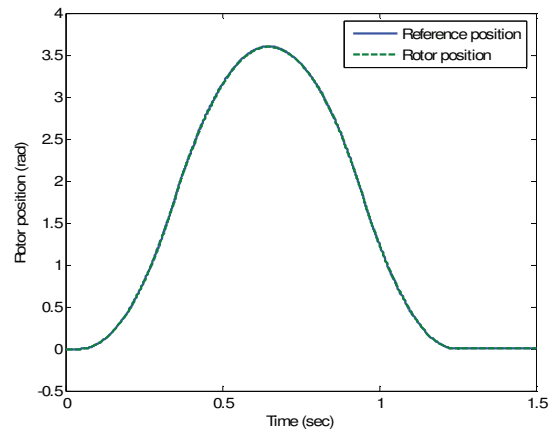

Fig. 15 Rotor position signals

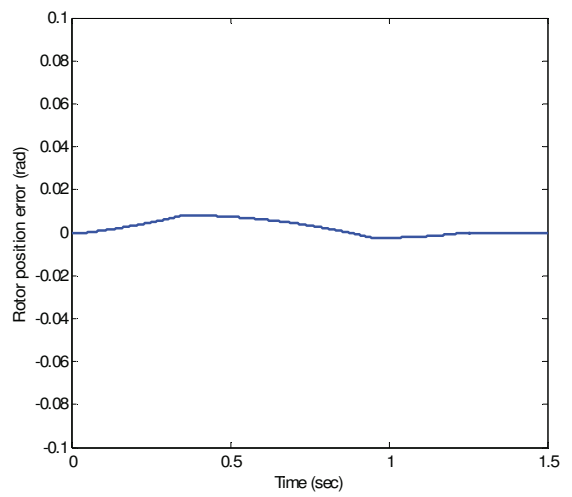

Fig. 16 Rotor position error

D. Results Comparison

The results show that the RLS algorithm, by considering the convergence time and the accuracy of the converged model is superior to the other introduced adaptive algorithms. Comparing Figure 7, Figure 11, and Figure 15, it can be concluded that high precision rotor position tracking performance can be achieved using the three adaptive algorithms. However, the RLS algorithm gives smaller peak rotor position error compared to the standard LMS and NLMS algorithms. This means that the RLS algorithm can track the rotor position command more accurately than the conventional LMS and NLMS algorithms. Robustness of the three adaptive algorithms against measurement noise is also verified. All three types of adaptive algorithms exhibit small sensitive to the measurement noise. However, the RLS algorithm still gives better convergence time compared to the other introduced algorithms.

\section{CoNCLUSIONS}

In this paper, a new methodology AIC is submitted to design the rotor position control of induction motor. To validate the performances of the new proposed control technique, we provided a series of simulation results and a comparative study between the LMS, NLMS and the RLS adaptive algorithms. Simulation results show that the RLS algorithm shows better performance than the other two adaptive algorithms.

\section{APPENDIX}

Table I Electrical and mechanical parameters of the IM

\begin{tabular}{|l|c|}
\hline \multicolumn{1}{|c|}{ Parameters } & Values \\
\hline Number of phases & 3 \\
\hline Connection & star \\
\hline Rated power & $2.24 \mathrm{KW}$ \\
\hline Line voltage & $230 \mathrm{~V} \mathrm{rms}$ \\
\hline Line current & $9 \mathrm{~A} \mathrm{rms}$ \\
\hline Rated speed & $1430 \mathrm{rpm}$ \\
\hline Rated torque & $14.96 \mathrm{Nm}$ \\
\hline Rotor resistance, $\mathrm{R}_{\mathrm{r}}$ & $0.72 \Omega$ \\
\hline Stator resistance, $\mathrm{R}_{\mathrm{s}}$ & $0.55 \Omega$ \\
\hline Rotor inductance, $\mathrm{L}_{\mathrm{r}}$ & $0.068 \mathrm{H}$ \\
\hline Stator inductance, $\mathrm{L}_{\mathrm{s}}$ & $0.068 \mathrm{H}$ \\
\hline Magnetising inductance, $\mathrm{L}_{\mathrm{m}}$ & $0.063 \mathrm{H}$ \\
\hline Moment of inertia, J & $0.05 \mathrm{~kg} \cdot \mathrm{m}^{2}$ \\
\hline Viscous friction coefficient, $\mathrm{B}$ & $0.002 \mathrm{Nms}^{-1}$ \\
\hline Number of pole pairs & 2 \\
\hline
\end{tabular}




\section{REFERENCES}

[1] Bose, B.K., 'Modern power electronics and ac drives', Pearson education, Inc., Indian, 2002

[2] S.Wade, M.W.Dunnigan, B.W.Williams, X.Yu, 'Position control of a vector controlled induction machine using slotine's sliding mode control', IEE Proceeding Electronics Power Application, Vol. 145, No.3, pp.231-238, 1998.

[3] S.J.Chapman, 'Electric Machinery Fundamentals', The McGraw-Hill Companies,

[4] Z.Beres, P.Vranka, 'Sensorless IFOC of induction motor with current regulators in current reference frame', IEEE Transactions on Industry Applications, Vol.37, pp.1012-1018, July 2001.

[5] F. Blaschke, 'The Principle of Field Orientation as Applied to the new Transvektor Closed-Loop Control System for Rotating Machines', Siemens Review, No. 34, 1972, pp217-220.

[6] R.J.Wai, K.H.Su, C.Y.Tu, 'Implementation of adaptive enhanced fuzzy sliding mode control for indirect field oriented induction motor drive', IEEE International Conference on Fuzzy Systems, pp.1440-1445, 2003.

[7] P.Vas, 'Vector control of ac machines', Oxford University Press, 1990.

[8] Trzynadlowski, 'The field oriented principle control of induction motors', Kluwer academic publishers, Massachusetts 02061 USA, 1994, pp813-818.

[9] B.Widrow, E.Walach, 'Adaptive Inverse Control', Prentice Hall Inc., NJ. 1996.

[10] B.Widrow, S.D.Stearns, 'Adaptive signal processing', Prentice-Hall, Englewood Cliffs, New Jersey, 1985.

[11] G.L.Plett, 'Adaptive inverse control of linear and nonlinear system using dynamic neural networks', IEEE Transactions on neural network, Vol.14, No.2, March 2003.

[12] S.Haykin, 'Adaptive filter theory', $4^{\text {th }}$ ed. Englewood Cliffs, New Jersey Prentice Hall, 2001

[13] J.Kim, E.T.Perry, 'Performance analysis of the self correcting adaptive filters', IEEE, pp.316-319, 2005.

[14] M.H.Hayes, 'Statistical digital signal processing and modelling', Jon Wiley and Sons, 1996.

[15] J.Kim, E.T.Perry, 'Performance analysis of the self correcting adaptive filters', IEEE, pp.316-319, 2005.

[16] R.R.Bitmead, B.D.O.Anderson, 'Performance of adaptive estimation algorithms in dependent random environments', IEEE Trans. On Automation Control, Vol. AC-25, pp.788-794, 1980.

[17] M.H.Hayes, 'Statistical digital signal processing and modelling', Jon Wiley and Sons, 1996.

[18] Y.Ahmed, F.Jawed, S.Zia, M.S.Aga, 'Real time implementation of adaptive channel equalization algorithms on TMS320C6x DSP processors', IEEE, 2004. 